\title{
El problema de Cauchy Local de un sistema dispersivo No Lineal de tipo Benjamin - Bona - Mahony
}

\author{
The Cauchy problem local System type nonlinear dispersive Benjamin - Bona - Mahony
}

\author{
Segundo Teófilo Vega Guadalupe ${ }^{1}$
}

\section{Resumen}

En el presente trabajo se estudia el sistema de ecuaciones no lineales dispersivas de tipo Benjamin-Bona-Mahony para la propagación de onda la cual incorpora efectos dispersivos. Nuestro objetivo es demostrar que el sistema dispersivo o problema de Cauchy está bien formulado localmente. Por esta razón se analiza varias propiedades de las soluciones $\mathrm{u}(\mathrm{x}, \mathrm{t}), \mathrm{v}(\mathrm{x}, \mathrm{t})$ para $\mathrm{x} \in \mathrm{IR}, \mathrm{t} \geq 0$.

Palabras clave: problema de Cauchy; sistema dispersivo no lineal BBM; buena formulación local.

\begin{abstract}
In this paper we study the system of nonlinear dispersive equations of the Benjamin-Bona-Mahony type for the propagation wave which incorporates dissipative effects. Our objective is to demonstrate that the nonlinear dispersive system, or Cauchy problem, is well formulated locally. This is why we analyze different properties of the solutions $\mathrm{u}(\mathrm{x}$ $, t), v(x, t)$ for $x \in I R, t \geq 0$.
\end{abstract}

Keywords: Cauchy problem; BBM nonlinear dispersive system; good local formulation.

\section{Introducción}

Consideremos una familia de ecuaciones dispersivas bajo el efecto de disipación

$\left(1-\partial_{x}^{2}\right) \partial_{t} u-a_{1} \partial_{x}^{2} \partial_{t} v+a_{2} \partial_{x} u+a_{3} v^{p} \partial_{x} v+a_{4} \partial_{x}\left(u^{p} v\right)+u^{p} \partial_{x} u=0$

$\left(1-\partial_{x}^{2}\right) \partial_{t} v-a_{1} \partial_{x}^{2} \partial_{t} u+a_{2} \partial_{x} v+a_{3} u^{p} \partial_{x} v+a_{4} \partial_{x}\left(u v^{p}\right)+v^{p} \partial_{x} v=0$

(P) $\mathrm{u}(0)=\mathrm{u} 0, \quad \mathrm{v}(0)=\mathrm{v} 0$

donde a1,a2,a3,a4 son constantes reales $\mathrm{a} 2>0,0<\mathrm{a} 1<1, \mathrm{u}=\mathrm{u}(\mathrm{x}, \mathrm{t}), \mathrm{v}=\mathrm{v}(\mathrm{x}, \mathrm{t}), \quad$ son funciones reales de variables reales $x$ y $t$ con $x \in R, t \geq 0$ y $p \geq 1$, $p$ un número entero. Aquí $\mathrm{u}_{\mathrm{x}}, \mathrm{v}_{\mathrm{x}}, \mathrm{u}_{\mathrm{t}}, \mathrm{v}_{\mathrm{t}}$ denotan derivadas parciales con respecto a $\mathrm{x}$ y $\mathrm{t}$ respectivamente. La existencia de las soluciones del sistema que describe la propagación de ondas largas de amplitud pequeña y finita en un canal de agua de profundidad constante, justifica la buena formulación local del problema de Cauchy.

Nuestro objetivo es estudiar las propiedades de las soluciones reales $\mathrm{u}(\mathrm{x}, \mathrm{t}), \mathrm{v}(\mathrm{x}, \mathrm{t})$ del problema de Cauchy (P), en el espacio de Sobolev $\mathbf{H}^{\mathrm{s}}(\mathbf{I R})=\mathrm{H}^{\mathrm{s}}(\mathrm{IR}) \times \mathrm{H}^{\mathrm{s}}(\mathrm{IR})$, cuya norma es dada por

$\|U\| \operatorname{Hs}(\mathrm{IR})=\|(u, v)\| \operatorname{Hs}(\mathrm{IR})=\sqrt{\|u\|_{H^{s}(I R)}^{2}+\|v\|_{\left.H^{s}(I R)\right)}^{2}}$, para $\mathrm{s} \geq 1$.

Demostraremos que $(\mathrm{P})$ está bien planteado localmente en el sentido de Hadamard, es decir:

P1. Existencia local de soluciones: Probar que existen $\mathrm{T} \epsilon] 0, \bar{T}]$ y UєC ([0,T];Hs(IR))tal que satisface (P).

P2. Unicidad: Probar que existe a lo más una solución de (P) en una vecindad del origen.

P3. Dependencia continúa del dato inicial: Estudiar y establecer, si es posible, la continuidad de la aplicación $\Phi \rightarrow U$ en topologías convenientes.

Se usará el teorema del punto fijo de Banach para probar que la ecuación integral asociada al sistema tiene solución y es única del problema de Cauchy (P).

\section{Materiales y métodos}

Preliminares

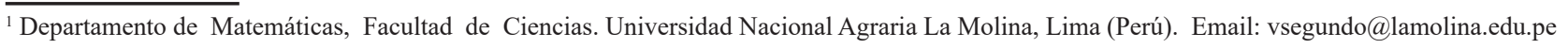


En esta sección se enuncian definiciones y propiedades básicas que usaremos en el trabajo. La prueba de los teoremas y proposiciones enunciados se pueden consultar en [2],[7],[8] y [9].

\section{Transformada de Fourier}

El espacio $L^{1}\left(\mathrm{IR}^{\mathrm{n}}\right)$ posee la multiplicación que lo convierte en un algebra de Banach.

Definición 1. Si u,v $\epsilon, L^{1}\left(I R^{n}\right)$ definimos su convolución por $\left(\mathrm{u}^{*} \mathrm{v}\right)=, \frac{1}{\sqrt{2 \pi}} \int_{I R^{n}} u(x-y) v(y) d y \quad \mathrm{x} \in \cdot I R^{n}$ La convolución es conmutativa y asociativa.

Teorema 2. (Desigualdad de Young). Si u $\epsilon L^{p}\left(I R^{n}\right)$ con $1 \leq \mathrm{p} \leq \infty$ y $\vee \in L^{1}\left(I R^{n}\right)$, entonces $\mathrm{u}^{*} \mathrm{v} \epsilon L^{p}\left(I R^{n}\right)$ y $\|u * v\|_{L^{p}} \leq\|u\|_{L^{p}}\|v\|_{L^{1}}$.

Teorema 3. Sean $1 \leq \mathrm{p}, \mathrm{q}, \mathrm{r} \leq \infty$ tales que $\frac{1}{p}+\frac{1}{q}=1+\frac{1}{r}$. Si u $\in L^{p}\left(I R^{n}\right)$ y $\vee \in L^{q}\left(I R^{n}\right)$, entonces $\mathrm{u}^{*} \mathrm{v} \in L^{r}\left(I R^{n}\right)$ y $\|u * v\|_{L^{r}} \leq\|u\|_{L^{p}}\|v\|_{L^{q}}$.

Proposición 4. Sean $\mathrm{k} \in L^{1}([a, b]), \mathrm{k}(\mathrm{t}) \geq 0$ para todo $\mathrm{t} \epsilon[\mathrm{a}, \mathrm{b}]$ y $f \in \mathrm{C}([\mathrm{a}, \mathrm{b}])$ tales que

$\mathrm{f}(\mathrm{t}) \leq \mathrm{g}(\mathrm{t})+\int_{a}^{t} k(s) f(s) d s, \mathrm{a} \leq \mathrm{t} \leq \mathrm{b}$, entonces

$\mathrm{f}(\mathrm{t}) \leq \mathrm{g}(\mathrm{t})+\int_{a}^{t} k(s) \exp \left[\int_{a}^{s} k(r) d r\right] g(s) d s, \mathrm{a} \leq \mathrm{t} \leq \mathrm{b}$.(1)

En particular si $\mathrm{g}(\mathrm{t})=\mathrm{C}=$ cte, se sigue que $\mathrm{f}(\mathrm{t}) \leq \mathrm{C} \exp$ $\left[\int_{a}^{t} k(s) d s\right], \mathrm{a} \leq \mathrm{t} \leq \mathrm{b}$.

Lema 5. Para cada $r \in I R$ existen constantes positivas $\mathrm{c}_{1}=\mathrm{c}_{1}(\mathrm{r}) \mathrm{y} \mathrm{c}_{2=} \mathrm{c}_{2}(\mathrm{r})$ tales que

$c_{1}\left(1+\xi^{2 r}\right) \leq\left(1+\xi^{2}\right)^{r} \leq c_{2}\left(1+\xi^{2 r}\right)$, para todo $\xi \in \mathrm{IR}$.

Transformada de Fourier en $L^{1}\left(I R^{n}\right)$

Definición 6. Sea u $€ L^{1}\left(I R^{n}\right)$ la transformada de

Fourier de $\mathrm{u}$, se define por

$\mathrm{Fu}(\xi)=\hat{u}(\xi)=\frac{1}{(2 \pi)^{n / 2}} \int_{I R^{n}} e^{-i\langle x, \xi\rangle} u(x) d x, \xi \in I R$

y la transformada inversa de Fourier de $\mathrm{u}$, como $\mathrm{F}^{-1} \mathrm{u}(\mathrm{x})=u(x)=\frac{1}{(2 \pi)^{n / 2}} \int_{I R^{n}} e^{i(x, \xi)} u(x) d x, \xi \in I R,\langle\mathrm{x}, \xi\rangle$ $=x_{1} \xi_{1}+\ldots .+x_{n} \xi_{n} \cdot(2 *)$

Teorema 7. Sean $u, v \in L^{1}\left(I R^{n}\right) y \alpha$ un número complejo. Entonces

1. La aplicación $\mathrm{F}: L^{1}\left(I R^{n}\right) \rightarrow L^{\infty}\left(I R^{n}\right)$ es una transformación lineal acotada y $\|F u\|_{L^{\infty}} \leq \frac{1}{(2 \pi)^{n / 2}}\|u\|_{L^{1}}$.

3. $\lim _{\xi \rightarrow 0} \mathbf{F u}(\xi)=0$ (Riemann Lebesgue).

4. $\mathrm{F}(\mathrm{u}+\mathrm{v})(\xi)=\mathrm{Fu}(\xi)+\mathrm{Fv}(\xi)$ y $\mathrm{F}(\alpha \mathrm{u})(\xi)=\alpha \mathrm{Fu}(\xi)$.

5. $\mathrm{F}\left(\mathrm{u}^{*} \mathrm{v}\right)(\xi)=(2 \pi)^{n / 2} F u(\xi) F v(\xi), \xi \in I R^{n}$.

Teorema 8. $\mathrm{Si} u \in L^{1}\left(I R^{n}\right)$ y $\partial^{\alpha} \mathrm{u} \in L^{1}\left(I R^{n}\right) \operatorname{con} \alpha \in \mathrm{N}$, entonces

$\mathrm{F}\left(\partial^{\alpha} \mathrm{u}\right)(\xi)=(\mathrm{i} \xi)^{\mathrm{n}} \mathrm{Fu}(\xi)$ si $\lim u(x)=0$.

Transformada de Fourier en $L^{2}\left(I R^{n}\right)$

Si u $\epsilon, L^{2}\left(I R^{n}\right), \hat{u}(\xi)=\frac{1}{(2 \pi)^{n / 2}} \int_{I R^{n}} e^{-i(x, \xi)} u(x) d x, \xi \in I R$ en general no tiene sentido. Para extender la transformada de Fourier a la transformada de Fourier en $L^{2}\left(I R^{n}\right)$ usaremos la propiedad de que el espacio $L^{1}\left(I R^{n}\right) \cap L^{2}\left(I R^{n}\right)$ es denso en $L^{2}\left(I R^{n}\right)$.

Teorema 9. Si u $\in L^{1}\left(I R^{n}\right) \cap L^{2}\left(I R^{n}\right)$, entonces Fu€ $L^{2}\left(I R^{n}\right)$ $\mathrm{y}\|F u\|_{L^{2}}=\|u\|_{L^{2}}$.

Por lo tanto F: $L^{1}\left(I R^{n}\right) \cap L^{2}\left(I R^{n}\right) \rightarrow L^{2}\left(I R^{n}\right)$ es un operador lineal acotado, así se tiene una única extensión lineal acotada, llamada transformada de Fourier sobre $L^{2}\left(I R^{n}\right)$

Teorema 10. La transformada de Fourier sobre $L^{2}\left(I R^{n}\right)$ es un operador lineal, isométrico y sobreyectivo.

Por lo tanto existe la transformada inversa de Fourier. ${ }^{v} L^{2}\left(I R^{n}\right) \rightarrow L^{2}\left(I R^{n}\right)$. Si

u $\in L^{2}\left(I R^{n}\right)$ y $\left\{u_{k}\right\}_{k \in N}$ es una sucesión en $L^{1}\left(I R^{n}\right) \cap L^{2}\left(I R^{n}\right)$

tal que la $\lim \left\|u_{k}-u\right\|_{L^{2}}=0$ transformada de Fourier de $\mathrm{u}$ en y $L^{2}\left(I R^{n}\right)$ la transformada inversa de Fourier de $\mathrm{u}$ en $L^{2}\left(I R^{n}\right)$ son calculadas por $\hat{u}(\xi)=\lim _{k \rightarrow+\infty} F u_{k}$ en $L^{2}\left(I R^{n}\right) \mathrm{y}$ $u(x)=\lim _{k \rightarrow+\infty} F^{-1} u_{k}$ en $L^{2}\left(I R^{n}\right)$.

\section{Distribución Temperada.}

El espacio de Schwartz es el conjunto de funciones reales $\mathrm{S}\left(\operatorname{IR}^{\mathrm{n}}\right)=\left\{\mathrm{u} \in \mathrm{C}^{\infty}\left(\operatorname{IR}^{\mathrm{n}}\right):\|\mathrm{u}\|_{\alpha, \beta}<+\infty\right.$, para todo $\alpha, \beta$ $\epsilon \mathrm{N}^{\mathrm{n}}$ \}, donde para cada par de multi-ìndices $(\alpha, \beta) \epsilon$ $\mathrm{N}^{\mathrm{n}} \mathrm{XN}^{\mathrm{n}}$, se tiene $\|u\|_{\alpha, \beta}=\sup _{x \in I R^{n}}\left|x^{\alpha} \partial_{x}^{\beta} u(x)\right|<+\infty$.

A la función $\mathrm{u}$ se le denomina función temperada y el espacio de Schwartz $S\left(I^{n}\right)$ es conocido como el espacio de las funciones $\mathrm{C}^{\infty}\left(\mathrm{IR}^{\mathrm{n}}\right)$ rápidamente decrecientes.

$\mathrm{S}\left(\mathrm{IR}^{\mathrm{n}}\right)$ con la métrica $d(u, v)=\sum_{\alpha, \beta} \frac{1}{2^{|\alpha|+|\beta|}} \frac{\|u-v\|_{\alpha, \beta}}{1+\|u-v\|_{\alpha, \beta}}$

es un espacio métrico completo.

Definición 11. La sucesión $\left\{u_{k}\right\}_{k \in N}$ en $\mathrm{S}\left(\mathrm{IR}^{\mathrm{n}}\right)$ converge a $u \in \mathrm{S}\left(\operatorname{IR}^{\mathrm{n}}\right)$ si $(\alpha, \beta) \in \mathrm{N}^{\mathrm{n}} \mathrm{XN}^{\mathrm{n}}: \lim _{k \rightarrow+\infty}\left\|u_{k}-u\right\|_{\alpha, \beta}=0$.

La relación entre la transformada de Fourier y el espacio $\mathrm{S}\left(\mathrm{IR}^{\mathrm{n}}\right)$ está descrita en el siguiente teorema.

Teorema 12. La transformada de Fourier es un isomorfismo de $\mathrm{S}\left(\mathrm{IR}^{\mathrm{n}}\right)$ en sí mismo.

Teorema 13. Sea $u \in S\left(I^{n}\right)$. Entonces

1. $\hat{u} \in S\left(I R^{n}\right)$.

2. $\partial^{\alpha} \hat{u}(\xi)=(-i)^{|\alpha|}\left(x^{\alpha} u\right)^{\wedge}(\xi)$ y $\left(\partial^{\alpha} u\right)^{\wedge}(\xi)=(i)^{|\alpha|} \xi^{\alpha} \hat{u}(\xi)$.

El dual lo definimos como el conjunto de las distribuciones temperadas.

Definición 14. T: $\mathrm{S}\left(\mathrm{IR}^{\mathrm{n}}\right) \rightarrow \mathrm{IR}$ define una distribución temperada, si $\mathrm{T}$ es una funcional lineal continua en $\mathrm{S}\left(\mathrm{IR}^{\mathrm{n}}\right)$, es decir, $\mathrm{T}$ es una distribución temperada si y solo si:

1. $\mathrm{T}$ es lineal.

2. $\mathrm{T}$ es continua con relación a la topología de $\mathrm{S}\left(\mathrm{IR}^{\mathrm{n}}\right)$, esto es, si $\mathrm{u}_{\mathrm{k}} \rightarrow \mathrm{u}$ en $\mathrm{S}\left(\mathrm{IR}^{\mathrm{n}}\right)$, entonces $\mathrm{T}\left(\mathrm{u}_{\mathrm{k}}\right) \rightarrow \mathrm{T}(\mathrm{u})$ en IR.

Así, $S^{\prime}\left(I R^{n}\right)$ denota el conjunto de las distribuciones temperadas.

Si T $\epsilon, S^{\prime}\left(I R^{n}\right)$ para u $\epsilon \mathrm{S}\left(\operatorname{IR}^{\mathrm{n}}\right)$ escribimos $\langle T, u\rangle=\langle T, u\rangle_{S^{\prime}, S}$ en vez de $\mathrm{T}(\mathrm{u})$. 
Definición 15. La función $u \in L_{l o c}^{1}\left(I R^{n}\right)$ es una distribución temperada si la funcional lineal $\mathrm{T}_{\mathrm{u}}$ : $\mathrm{S}\left(\mathrm{IR}^{\mathrm{n}}\right) \rightarrow \mathrm{IR}$ dado por $\mathrm{T}_{\mathrm{u}}(\mathrm{v})=\int_{I R} u(x) v(x) d x, v \in S\left(I R^{n^{\mathrm{u}}}\right)$ es continuo.

Teorema 16. Los elementos de $L^{p}\left(I R^{n}\right), 1 \leq \mathrm{p} \leq+\infty$, son distribuciones temperadas.

Se prueba que $L^{p}\left(I R^{n}\right)$ es continua y está densamente incluido en $S^{\prime}\left(I R^{n}\right)$ es decir, $L^{p}\left(I R^{n}\right) \cup \quad S^{\prime}\left(I R^{n}\right)$ si $1 \leq \mathrm{p}$ $\leq \infty$. Por lo tanto, identificaremos a $L^{p}\left(I R^{n}\right)$ como un subespacio de $S^{\prime}\left(I R^{n}\right)$. La distribución $\mathrm{T}_{\mathrm{u}} \in S^{\prime}\left(I R^{n}\right)$ será identificada con u y u $(\mathrm{v})=\langle u, v\rangle$, u $\in S^{\prime}\left(I R^{n}\right)$ y $\mathrm{v} \in \mathrm{S}\left(\mathrm{IR}^{\mathrm{n}}\right)$, en particular, si $\mathrm{u} \epsilon, L^{p}\left(I R^{n}\right) 1 \leq \mathrm{p} \leq \infty$, se escribe $\mathrm{u}(\mathrm{v})=\int_{I R} u(x) v(x) d x, v \in S\left(I R^{n}\right)$

Definición 17. Una distribución temperada $T \in S^{\prime}\left(I R^{n}\right)$ es una función en $L_{l o c}^{1}\left(I R^{n}\right)$

si $\mathrm{T}_{\mathrm{u}} \in S^{\prime}\left(I R^{n}\right)$ y $\mathrm{T}=\mathrm{T}_{\mathrm{u}}$.

Definición 18. (Derivada de una distribución). Dados $\mathrm{T}$ $\in S^{\prime}\left(I R^{n}\right)$ y $\alpha € N^{n}$ un multi-índice, definimos la $\alpha$-ésima derivada de $\mathrm{T}$ por $\left\langle\partial^{\alpha} T, v\right\rangle=(-1)^{|\alpha|}\left\langle T, \partial^{\alpha} v\right\rangle$, para cualquier $v \in S\left(I R^{n}\right)$. Además, $\partial^{\alpha} T \in S^{\prime}\left(I R^{n}\right)$.

\section{Transformada de Fourier en $S^{\prime}\left(I R^{n}\right)$}

Definiremos la transformada de Fourier en $S^{\prime}\left(I R^{n}\right)$ vía la transformada de Fourier en $S^{\prime}\left(I R^{n}\right)$.

Definición 19. Si u $\in S^{\prime}\left(I R^{n}\right)$, la transformada de Fourier y la transformada inversa de Fourier de $\mathrm{u}$ se define por $\langle\hat{u}, v\rangle=\langle u, \hat{v}\rangle$ y $\langle u, v\rangle=\langle u, v\rangle$, para $\mathrm{v} \epsilon$ $S\left(I R^{n}\right)$.

La topología en $S^{\prime}\left(I R^{n}\right)$ está determinada de la manera siguiente.

Definición 20. Sea $\left\{\mathrm{u}_{\mathrm{k}}\right\}_{\mathrm{keN}}$ una sucesión en $S^{\prime}\left(I R^{n}\right)$. Decimos que $u_{\mathrm{k}} \rightarrow 0$ en $S\left(I R^{n}\right)$ si $\mathrm{k} \rightarrow+\infty$, es decir, para todo $v \in S\left(I R^{n}\right)^{k}:\left\langle u_{k}, v\right\rangle \rightarrow 0$ cuando $\mathrm{k} \rightarrow+\infty$.

Como consecuencia resulta el teorema de extensión.

Teorema 21. La transformada de Fourier es un isomorfismo de $S^{\prime}\left(I R^{n}\right)$ en sí mismo, es decir, ${ }^{\wedge}: S^{\prime}\left(I R^{n}\right)$ $\rightarrow S^{\prime}\left(I R^{n}\right)$ es biyectiva, continua con inversa continua.

Proposición 22. Si u $\in S^{\prime}\left(I R^{n}\right)$, entonces $\left(\partial^{\alpha} u\right)^{\wedge}=(i)^{|\alpha|} \xi^{\alpha} \hat{u}$ y $\partial^{\alpha} \hat{u}=(-i)^{|\alpha|}\left(x^{\alpha} u\right)^{\wedge}$.

Definición 23. Para $\mathrm{s} \in \mathrm{IR}$ definimos el operador lineal $J^{s}: S^{\prime}\left(I R^{n}\right) \rightarrow S^{\prime}\left(I R^{n}\right)$ por $\left(J^{s} u \hat{)}=\left(1+|\xi|^{2}\right)^{s / 2} \hat{u}(\xi), \mathrm{u} \epsilon\right.$

$S^{\prime}\left(I R^{n}\right)$ denominado el potencial de Bessel de orden $\mathrm{s}$. $J^{s}$ es una aplicación lineal, continua y biyectiva.

Espacios de Sobolev de tipo $L^{2}\left(I R^{n}\right)$

Daremos una breve introducción a los espacios de Sobolev de orden $\mathrm{s} \in \mathrm{IR}$ en $I R^{n}$, a través de la transformada de Fourier en $S^{\prime}\left(I R^{n}\right)^{\prime}$. Ellos miden la diferenciabilidad de las funciones en $L^{2}\left(I R^{n}\right)$ y son de gran ayuda en el estudio de las ecuaciones en derivadas parciales.

Definición 24. El espacio de Sobolev de orden s $€$ IR de tipo $L^{2}\left(I R^{n}\right)$ es el conjunto $H^{s}\left(I R^{n}\right)$, definido por
$H^{s}\left(I R^{n}\right)=\left\{u \in S^{\prime}\left(I R^{n}\right): J^{s} u \in L^{2}\left(I R^{n}\right)\right\}$,

Con norma $\|u\|_{H^{s}\left(I R^{n}\right)}=\left\|J^{s} u\right\|_{L^{2}\left(I R^{n}\right)}=\left(\int_{I R^{n}}\left(1+|\xi|^{2}\right)^{s}|\hat{u}(\xi)|^{2} d \xi\right)^{1 / 2}$.

En particular $=$. Usaremos la notación en vez de.

\section{Teorema 25.}

1. Si $0 \leq \mathrm{s} \leq \mathrm{t}$ entonces $H^{t}\left(I R^{n}\right) \smile H^{s}\left(I R^{n}\right)$ es densamente continua. Además para todo u $\epsilon: H^{s}\left(I R^{n}\right)$ $\|u\|_{s} \leq\|u\|_{t}$. En particular, los elementos de $\|u\|_{t}$ para $\mathrm{s} \geq 0$ son funciones medibles, más precisamente, son distribuciones temperadas que provienen de funciones en $L^{2}\left(I R^{n}\right)$.

2. $H^{s}\left(I R^{n}\right)$ es un espacio de Hilbert separable con el producto interno definido

para todo $\mathrm{u}, \mathrm{v} \in H^{s}\left(I R^{n}\right)$ por

$\langle u, v\rangle_{s}=\left\langle J^{s} u, J^{s} v\right\rangle_{L^{2}\left(I R^{n}\right)}=\int_{I R^{n}}\left(1+|\xi|^{2}\right)^{s} \hat{u}(\xi) \overline{\hat{v}}(\xi) d \xi$

es decir, vía la transformada de Fourier $H^{s}\left(I R^{n}\right)=$ $L^{2}\left(I R^{n}:\left(1+|\xi|^{2}\right)^{s} d \xi\right)$.

3. Para todo $\mathrm{S} \in \mathrm{IR}$ el espacio $\mathrm{S}\left(\mathrm{IR}^{\mathrm{n}}\right)$ es denso en $H^{s}\left(I R^{n}\right)$.

4. Para todo $\mathrm{k} \in \mathrm{N}$ y para todo $\mathrm{s} \in \mathrm{IR}, \mathrm{D}^{\mathrm{k}}$ es un operador acotado en $H^{s}$ hacía $H^{s-k}(I R)$. Además, $\left\|D^{k} u\right\|_{H^{s-k}} \leq c\|u\|_{H^{s}}$.

5. Sea $\mathrm{s}>\mathrm{n} / 2, \mathrm{~s} \in \mathrm{IR}$. Entonces $H^{s}(I R)$ es un algebra conmutativa en relación a las operaciones de multiplicación de funciones punto a punto y para cualquier $\mathrm{u}, \mathrm{v} \in H^{s}(I R)$, existe una constante $\mathrm{c}=\mathrm{c}(\mathrm{s}, \mathrm{n})$ tal que $\|u v\|_{s} \leq c\|u\|_{s}\|v\|_{s}$.

Teorema 26. (Inmersión de Sobolev). Si s > $1 / 2+\mathrm{k}$ entonces $H^{s}(I R)$ está contenido continuamente en el espacio $C_{\infty}^{k}\left(I R^{n}\right)$ de las funciones con $\mathrm{k}$ derivadas continuas que se anulan en el infinito,

$\mathrm{y}\|u\|_{C_{\infty}^{k}} \leq c_{s}\|u\|_{s}$. En consecuencia, si $\mathrm{n}=1$ y $\mathrm{s}>1 / 2+\mathrm{k}$, $\|u\|_{L^{\infty}} \leq c_{s}\|u\|_{s},\left\|\partial_{x} u\right\|_{L^{\infty}} \leq c_{s}\|u\|_{s},\left\|\partial_{x}^{2} u\right\|_{L^{\infty}} \leq c_{s}\|u\|_{s}, \ldots$.

\section{El problema de Cauchy local}

El problema de Cauchy $(\mathrm{P})$ es equivalente a $\mathrm{A} \partial_{\mathrm{t}} \mathrm{U}(\mathrm{t})+\mathrm{B} \partial_{\mathrm{x}} \mathrm{U}(\mathrm{t})+\partial_{\mathrm{x}} \mathrm{F}(\mathrm{U}(\mathrm{t}))=0, \mathrm{U}(0)=\left(\mathrm{u}_{0}, \mathrm{v}_{0}\right)(3)$

donde $\mathrm{A}=\left(\begin{array}{cc}1-\partial_{x}^{2} & -a_{1} \partial_{x}^{2} \\ -a_{1} \partial_{x}^{2} & 1-\partial_{x}^{2}\end{array}\right), \mathrm{B}=\left(\begin{array}{cc}a_{2} & 0 \\ 0 & a_{2}\end{array}\right)$,

$\mathrm{FU}=\left(\begin{array}{c}\frac{a_{3}}{p+1} v^{p+1}+a_{4}\left(u^{p} v\right)+\frac{1}{p+1} u^{p+1} \\ \frac{a_{3}}{p+1} u^{p+1}+a_{4}\left(u v^{p}\right)+\frac{1}{p+1} v^{p+1}\end{array}\right)$

De la ecuación (4), obtenemos $\mathrm{U}(\mathrm{x}, \mathrm{t})=\mathrm{U}_{0}(\mathrm{x})$ $\int_{0}^{t} A^{-1}\left(B \partial_{x} U(x, \tau)+\partial_{x} F(U(x, \tau))\right) d \tau$, para justificar esta igualdad, analizamos la existencia del operador.

Teorema 27. Si $0<\mathrm{a}_{1}<1$ y s $\geq 2$, entonces A:

$H^{s} x H^{s} \rightarrow H^{s-2} x H^{s-2}$ es un operador lineal, simétrico, biyectivo y para $\mathrm{g}=\left(\mathrm{g}_{1}, \mathrm{~g}_{2}\right) \in H^{s-2} x H^{s-2}$ se tiene 
$\mathrm{g}(\mathrm{x})=\mathrm{K} * \mathrm{~g}(\mathrm{x})(5)$ donde $\mathrm{K}=\left(\mathrm{K}_{\mathrm{lr}}\right)_{1, \mathrm{l}=1,2} \mathrm{y} \mathrm{K}_{\mathrm{lr}}(\mathrm{x})=$

$\frac{1}{\sqrt{2 \pi}} \int_{I R} e^{i \xi x} a_{l r}(\xi) d \xi, \operatorname{con}\left(A^{-1}\right)(\xi)=\left(a_{l r}(\xi)\right)_{l, r=1,2}$.

Además, $K_{l r} \in L^{1} \cap L^{\infty}$ para $1, \mathrm{r}=1,2$.

Demostración. La linealidad del operador A es inmediata. Probemos que Af $\epsilon H^{s-2} x H^{s-2}$. En efecto, sea $\mathrm{f}=\left(\mathrm{f}_{1}, \mathrm{f}_{2}\right) \in H^{s} x H^{s}$ con $\mathrm{s} \geq 2$.

$\|A f\|_{H^{s-2} x H^{s-2}}=\left\|J^{2} f_{1}-a_{1} \partial_{x}^{2} f_{2}\right\|_{s-2}^{2}+\left\|-a_{1} \partial_{x}^{2} f_{1}+J^{2} f_{2}\right\|_{s-2}^{2}$.

Por la desigualdad de Cauchy-Schwartz, la inclusión continua del espacio de Sobolev de $H^{s}$ en $H^{s-2}$ y el hecho de que el operador $\partial_{x}: H^{s-1} \rightarrow H^{s-2}$ es acotado, tenemos $\|A f\|_{H^{s-2} x H^{s-2}} \leq\left(1+\left|a_{1}\right|^{2}\right)\|f\|_{H^{s} x H^{s}}^{2}<\infty$, entonces $\mathrm{R}(\mathrm{A}) \subseteq H^{s-2} x H^{s-2} \quad$ Probemos la inyectividad del operador A. Para $\mathrm{U}=(\mathrm{u}, \mathrm{v}) \in H^{s} x H^{s}, \mathrm{~s} \geq 2$, usando integración por partes y la inclusión continua tenemos $\langle A U, U\rangle_{L^{2} x L^{2}} \geq\|u\|_{L^{2}}^{2}+\left(1-\left|a_{1}\right|\right)\left\|\partial_{x} u\right\|_{L^{2}}^{2}+\|v\|_{L^{2}}^{2}+\left(1-\left|a_{1}\right|\right)\left\|\partial_{x} v\right\|_{L^{2}}^{2}$. De $\left|a_{1}\right|<1$ se tiene $\left(1-\left|a_{1}\right|\right)>0$, por lo tanto $\langle A U, U\rangle_{L^{2} \times L^{2}} \geq\|u\|_{L^{2}}^{2}+$ $\|v\|_{L^{2}}^{2}=\|U\|_{L^{2} x L^{2}}^{2} \geq 0$. En base a esto se prueba que el operador A es inyectivo. En efecto, sea $\mathrm{AU}=\mathrm{AV}$, entonces $\mathrm{A}(\mathrm{U}-\mathrm{V})=0$. Así $0=\langle A(U-V), U-V\rangle_{L^{2} x L^{2}} \geq\|U-V\|_{L^{2} x L^{2}}^{2}$ luego el núcleo $\operatorname{ker}(\mathrm{A})=\{0\}$. De ahí que existe .

Probaremos que el operador A es simétrico. En efecto, sea $\mathrm{U}=\left(\mathrm{u}_{1} \mathrm{u}_{2}\right), \mathrm{V}=\left(\mathrm{v}_{1}, \mathrm{v}_{2}\right) \in H^{s}\left(I R^{n}\right), \mathrm{s} \geq 2$, se tiene $\langle A U, V\rangle_{L^{2} x L^{2}}=\left\langle u_{1}, v_{1}\right\rangle_{L^{2}}+\left\langle u_{1}, v_{1}\right\rangle_{L^{2}}+\left\langle-\partial_{x}^{2} u_{1}, v_{1}\right\rangle_{L^{2}}+\left\langle-a_{1} \partial_{x}^{2} u_{2}, v_{1}\right\rangle_{L^{2}}$ $+\left\langle-a_{1} \partial_{x}^{2} u_{1}, v_{2}\right\rangle_{L^{2}}+\left\langle u_{2}, v_{2}\right\rangle_{L^{2}}+\left\langle-\partial_{x}^{2} u_{2}, v_{2}\right\rangle_{L^{2}}$.

Usando la integración por partes y la inclusión continua obtenemos $\langle A U, V\rangle_{L^{2} \times L^{2}}=\langle A U, V\rangle_{L^{2} x L^{2}}$, entonces $\mathrm{A}$ es simétrica. Que A es sobreyectiva, dado $\mathrm{g}=\left(\mathrm{g}_{1}, \mathrm{~g}_{2}\right) \mathrm{E}$ $H^{s-2} x H^{s-2}$ existe $\mathrm{f}=\left(\mathrm{f}_{1}, \mathrm{f}_{2}\right) \in H^{s} x H^{s}$ tal que $\mathrm{Af}=\mathrm{g}$. En efecto, de $\mathrm{A}\left(\mathrm{f}_{1}, \mathrm{f}_{2}\right)=\left(\mathrm{g}_{1}, \mathrm{~g}_{2}\right)$ se tiene que $\left(1-\partial_{x}^{2}\right) f_{1}-a_{1} \partial_{x}^{2} f_{2}=g_{1} ;\left(1-\partial_{x}^{2}\right) f_{2}-a_{1} \partial_{x}^{2} f_{1}=g_{2}$

Luego, aplicando la transformąda de Føurier respecto de la variable espacial, $\left(1+\xi^{2}\right) f_{1}(\xi)+a_{1} f_{2}(\xi)=\hat{g}_{1}(\xi)$; de donde $\hat{f}_{1}(\xi)=\frac{-a_{1} \xi^{2} \hat{g}_{2}(\xi)+\left(1+\xi^{2}\right) \hat{g_{1}}(\xi)}{\left(1+\xi^{2}\right)^{2}-a_{1}^{2} \xi^{4}}$; $\hat{f}_{2}(\xi)=\frac{-a_{1} \xi^{2} \hat{g}_{1}(\xi)+\left(1+\xi^{2}\right) \hat{g}_{2}(\xi)}{\left(1+\xi^{2}\right)^{2}-a_{1}^{2} \xi^{4}}$ Luego, para $g=\left(\mathrm{g}_{1,} \mathrm{~g}_{2}\right)$, $\int_{I R}\left(1+\xi^{2}\right)^{s}\left|\frac{-a_{1} \xi^{2} \hat{\xi}_{2}(\xi)+\left(1+\xi^{2}\right) \hat{g}_{1}(\xi)}{\left(1+\xi^{2}\right)^{2}-a_{1}^{2} \xi^{4}}\right|^{2} d \xi+\int_{I R}\left(1+\xi^{2}\right)^{s}\left|-\frac{a_{1} \xi^{2} \hat{\xi}_{1}(\xi)+\left(1+\xi^{2}\right) \hat{g}_{2}(\xi)}{\left(1+\xi^{2}\right)^{2}-a_{1}^{2} \xi^{2}}\right|^{2} d \xi$

Vía transformada de Fourier, se tiene

$$
\left(A^{-1} g\right)^{\wedge}(\xi)=\left(\begin{array}{ll}
\frac{1+\xi^{2}}{\left(1+\xi^{2}\right)^{2}-a_{1}^{2} \xi^{4}} & \frac{-a_{1} \xi^{2}}{\left(1+\xi^{2}\right)^{2}-a_{1}^{2} \xi^{4}} \\
\frac{-a_{1} \xi^{2}}{\left(1+\xi^{2}\right)^{2}-a_{1}^{2} \xi^{4}} & \frac{1+\xi^{2}}{\left(1+\xi^{2}\right)^{2}-a_{1}^{2} \xi^{4}}
\end{array}\right)\left(\begin{array}{l}
\hat{g}_{1}(\xi) \\
\hat{g}_{2}(\xi)
\end{array}\right)
$$

$=\left(a_{l r}(\xi)\right) \hat{g}(\xi)=\hat{K}_{l r}(\xi) \hat{g}(\xi)=(K * g)^{\wedge}(\xi)$

De donde se concluye que y por definición de norma en se verifica que $A^{-1} g \in H^{s} x H^{s}$ siempre que $\mathrm{g}=\left(\mathrm{g}_{1}, \mathrm{~g}_{2}\right)$ є $H^{s-2} x H^{s-2}$.

De la definición de $\mathrm{K}_{\mathrm{lr}}(\mathrm{x})$ :

$$
\begin{aligned}
& K_{11}(x)=K_{22}(x)=\frac{1}{\sqrt{2 \pi}} \int_{I R} e^{i \xi x} \frac{1+\xi^{2}}{\left(1+\xi^{2}\right)^{2}-a_{1}^{2} \xi^{4}} d \xi \\
& K_{12}(x)=K_{21}(x)=\frac{1}{\sqrt{2 \pi}} \int_{I R} e^{i \xi x} \frac{a_{1} \xi^{2}}{\left(1+\xi^{2}\right)^{2}-a_{1}^{2} \xi^{4}} d \xi \text {. Del lema 5, } \\
& \left|K_{11}(x)\right| \leq \frac{1}{\sqrt{2 \pi}} \int_{I R} \frac{1+\xi^{2}}{\left(1+\xi^{2}\right)^{2}-a_{1}^{2} \xi^{4}} d \xi \leq \frac{1}{\sqrt{2 \pi}} \int_{I R} \frac{C}{1+\xi^{2}} \leq C \sqrt{\frac{\pi}{2}}
\end{aligned}
$$

Así, $K_{11} \in L^{\infty}$. Por demostrar que . En efecto, $\int_{I R}\left|K_{11}(x)\right| d x=\int_{|x| \leq \mid}\left|K_{11}\right| d x+\int_{|x| 1 \mid}\left|K_{11}\right| d x \quad$, donde la primera integral del segundo miembro existe. De la definición de $\mathrm{K}_{11}$ para $\mathrm{x}=0, \mathrm{~K}(0)=$ existe. Para $\mathrm{x} \neq 0$, integrando por partes $\mathrm{K}_{11}(\mathrm{x})=\mathrm{d} \xi$-d $\xi$

Donde $\mathrm{u}(\xi) \mathrm{v}(\xi)=[\mathrm{u}(\xi) \mathrm{v}(\xi)-\mathrm{u}(-\xi) \mathrm{v}(-\xi)]$, como $0<\mathrm{a}_{1}$ $\prec 1$, los coeficientes de cada término del numerador están acotados. Luego, existe $\mathrm{C}>0$ tal que

$\left|K_{11}\right| \leq \frac{C}{x^{2}} \int_{I R} \frac{1+|\xi|^{2}+|\xi|^{4}+|\xi|^{6}+|\xi|^{8}}{\left[1+\left(1-a_{1}^{2}\right) \xi^{4}\right]^{3}} d \xi$

pues $\left(1+\xi^{2}\right)^{2}-a_{1}^{2} \xi^{4} \geq 1+\left(1-a_{1}^{2}\right) \xi^{4}$. Teniendo en cuenta que $\left[1+\left(1-a_{1}^{2}\right)^{\frac{1}{4}}|\xi|\right]^{4} \leq C\left[1+\left.\left.\left(1-, a_{p}^{2}\right)|t| \xi\right|^{4}\right|^{4}<\mathrm{a}_{1}<1\right.$, en (7)

se tiene $\left|K_{11}(x)\right| \leq \frac{C}{x^{2}} \int_{I R} \frac{1+|\xi|^{2}+|\xi|^{4}+|\xi|^{6}+|\xi|^{8}}{\left[1+\left.\left(1-a_{1}^{2}\right)^{\frac{1}{4}}|\xi|\right|^{12}\right.} d \xi$ para $\mathrm{n}=2,4,6,8$, se cumple $|\xi|^{n} \leq C\left[1+\left(1-a_{1}^{2}\right)^{\frac{1}{4}}|\xi|\right]^{n}$
y para $\mathrm{t}=4,6,8,10,12$

la integral $\int_{0}^{\infty} \frac{d \xi}{(1+\xi)^{t}} d x=\int_{1}^{\infty} \frac{d x}{u^{t}}$ onverge; en (7) se tiene que $\left|K_{11}\right| \leq \frac{C}{x^{2}}$. De donde $\int_{|x| \geq \mid}\left|K_{11}(x)\right| d x \leq C \int_{|x| x \mid 1} \frac{d x}{x^{2}}<\infty$. Por consiguiente, $K_{11} \in L^{1}$, así $K_{11}=K_{22} \in L^{1} \cap L^{\infty}$ De igual manera se prueba que $K_{12}=K_{21} \in L^{1} \cap L^{\infty}$

Teorema 28. Sea $\mathrm{g}=\left(\mathrm{g}_{1}, \mathrm{~g}_{2}\right) \in H^{s} x H^{s}, \mathrm{~s} \in \mathrm{IR}, \mathrm{s} \geq 1$ y $0<$ $\mathrm{a}_{1}<1$. Entonces $\left(\mathrm{K} . \partial_{\mathrm{x}} \mathrm{g}\right) \in H^{s} x H^{s}$ y existe una constante positiva $C$ tal que $\left\|K^{*} \partial_{x} g\right\|_{H^{s} x H^{s}} \leq C\|g\|_{H^{s} x H^{s}}$.

Demostración. De (6) y del teorema 27 tenemos que $\left(\mathrm{K}^{*} \partial_{\mathrm{x}} \mathrm{g}\right)(\mathrm{x})=\left(\left(\mathrm{a}_{11}\right)^{\sim} \partial_{\mathrm{x}} \mathrm{g}_{1}(\mathrm{x})+\left(\mathrm{a}_{12}\right)^{\sim} \partial_{\mathrm{x}} \mathrm{g}_{2}(\mathrm{x}),\left(\mathrm{a}_{21}\right)^{\sim} \partial_{\mathrm{x}} \mathrm{g}_{1}(\mathrm{x})+\right.$ $\left.\left(\mathrm{a}_{22}\right) \partial_{\mathrm{x}} \mathrm{g}_{2}(\mathrm{x})\right)$. Aplicando la definición de norma en el espacio de Sobolev, el lema 5 y teniendo en cuenta que $0<a_{1}<1$, se tiene

$\left\|K^{*} \partial_{x} g\right\|_{H^{x} x H^{5}}^{2} \leq C \int_{I R}\left(1+\xi^{2}\right)^{s}\left|\frac{\left(1+\xi^{2}\right) \xi}{\mid\left(1+\xi^{2}\right)^{2}-a_{1}^{2} \xi^{4}}\right|^{2}\left(\left|\hat{g}_{1}(\xi)\right|^{2}+\left|\hat{g}_{2}(\xi)\right|^{2}\right) d \xi$

Pero $\mid \frac{\left(1+\xi^{2}\right) \xi}{\left(1+\xi^{2}\right)^{2}-a_{1}^{2} \xi^{4}}<C$, para $C>0$,

$\left\|K^{*} \partial_{x} g\right\|_{H^{s} x H^{s}}^{2} \leq C \int_{I R}\left(1+\xi^{2}\right)^{s}\left(\left|\hat{g}_{1}(\xi)\right|^{2}+\left|\hat{g}_{2}(\xi)\right|^{2}\right) d \xi=\|g\|_{H^{s} x H^{s}}^{2}$ $\mathrm{C}<\infty$. Por lo tanto $\left(\mathrm{K} \cdot{ }^{*} \partial_{\mathrm{x}} \mathrm{g}\right) \in H^{s} x H^{s}$.

\section{Existencia Local de Soluciones}

Se probará que existe $\left.\mathrm{T} \epsilon] 0, \mathrm{~T}_{0}\right]$ y $(\mathrm{u}, \mathrm{v}) \in C\left(\left[0, T_{0}\right]: H^{s} x H^{s}\right)$ tal que satisface (3). La derivada respecto al tiempo 
debe ser interpretada en el sentido de la topología de $H^{s}$. La definición de existencia local posee la propiedad de persistencia de la solución, es decir, la solución en cuanto existe, permanece en el espacio de $H^{s}$.

Proposición 29. Sean $\mathrm{U}(0)=(\mathrm{u}, \mathrm{v}) H^{s} x H^{s} \epsilon, \mathrm{s} \geq 2, \mathrm{~s} \in \mathrm{N}$, $\mathrm{a}_{1}, \mathrm{a}_{2}, \mathrm{a}_{3}, \mathrm{a}_{4}$ números reales con $\mathrm{a}_{2}>0,0<\mathrm{a}_{1}<1 \mathrm{y} \mathrm{p} \geq 1$. $\mathrm{Si}(\mathrm{u}, \mathrm{v}) \in C\left(\left[0, T_{0}\right]: H^{s} x H^{s}\right)$ es una solución del problema (4), entonces $\mathrm{U}(\mathrm{x}, \mathrm{t})=\mathrm{U}_{0}(\mathrm{x})-\int_{0}^{t} A^{-1}\left[B \partial_{x} U(x, \tau)+\partial_{x} F(U(x, \tau))\right]$ (EI)

Demostración. Para $\mathrm{t}>0, \mathrm{t} \in \mathrm{IR}$, como $(\mathrm{u}(\mathrm{t}), \mathrm{v}(\mathrm{t}))$ es solución de (4), $\mathrm{U}(\mathrm{t})=(\mathrm{u}(\mathrm{t}), \mathrm{v}(\mathrm{t}))$ satisface la ecuación $\mathrm{A} \partial \mathrm{t} \mathrm{U}(\mathrm{t})+\mathrm{B} \partial \mathrm{x} \mathrm{U}(\mathrm{t})+\partial \mathrm{x} \mathrm{F}(\mathrm{U}(\mathrm{t}))=0$, por el teorema 27 existe $A^{-1}$, integrando de 0 a t, resulta $\mathrm{U}(\mathrm{t})=\mathrm{U}(0)$ $-\int_{0}^{t} A^{-1}\left[B \partial_{x} U(\tau)+\partial_{x} F(U(\tau))\right]$ Esto significa que si $\mathrm{U} \epsilon$ $C\left([0, T]: H^{s}\right)^{2}$ es solución de (3), también es solución de (EI). Nos preguntamos, ¿toda solución de (EI) es solución de (3)?, para dar respuesta a esta pregunta necesitamos el teorema del punto fijo de Banach. Para esto consideremos el espacio métrico completo definido por el espacio de funciones.

$\mathrm{Y}(\mathrm{T}, \mathrm{R})=\left\{\mathrm{U}=(\mathrm{u}, \mathrm{v}) \epsilon_{C}\left([0, T]: H^{s}\right)^{2}: \sup _{t \in[0, T]}\|U(t)-U(0)\|_{H^{s} x H^{s}} \leq R\right\}$ La norma en $\mathrm{Y}(\mathrm{T}, \mathrm{R})$ es dada por $\|U\|_{Y(T, R)}^{t \in[, T]}, \sup _{t \in[0, T}\|U(., t)\|_{H^{s} x H^{s}}$, donde $\mathrm{s} \geq 2,\left(\mathrm{u}_{0}, \mathrm{v}_{0}\right) \in H^{s} x H^{s}, \mathrm{~T}$ y $\mathrm{R}$ reales positivos. Es claro que $(\mathrm{Y}(\mathrm{T}, \mathrm{R}), \mathrm{d})$ es un espacio métrico completo. Para $\mathrm{U} \in \mathrm{Y}(\mathrm{T}, \mathrm{R})$, definimos la aplicación $\mathrm{P}$ por $(\mathrm{PU})$ $(\mathrm{x}, \mathrm{t})=\mathrm{U}(\mathrm{x}, 0)-\int_{0}^{t} A^{-1}\left[B \partial_{x} U(x, \tau)+\partial_{x} F(U(x, \tau))\right] d \tau, \forall t \in[0, T]$, (9)

Definido el espacio $\mathrm{Y}(\mathrm{T}, \mathrm{R})$ y la aplicación $\mathrm{P}$ se tiene la siguiente proposición.

Proposición 30. Si $\mathrm{s} \geq 2$ y U(.,0) $H^{s} x H^{s}$, con U(.,0) $\neq 0$ existen $\bar{T} y \bar{R}$ positivos dependientes de $\|U(., 0)\|_{H^{s} x H^{s}}$, tales que $P: Y(\bar{T}, \bar{R}) \rightarrow Y(\bar{T}, \bar{R})$ es una contracción.

Demostración.Probaremos que la aplicación P satisface el siguiente esquema $P: Y(\bar{T}, \bar{R}) \rightarrow Y(\bar{T}, \bar{R}) \subset C\left([0, \bar{T}]: H^{s} x H^{s}\right)$

$\mathrm{U} \rightarrow \mathrm{PU}:[0, \bar{T}] \rightarrow H^{s} x H^{s}$

$\mathrm{t} \rightarrow \mathrm{PU}(\mathrm{t})$

y lo haremos en dos etapas.

Primera etapa.

Probaremos que $\mathrm{P}$ tiene rango $\mathrm{R}(\mathrm{P}) \subseteq Y(\bar{T}, \bar{R})$ para $\bar{T} y \bar{R}$ positivos dependientes de $\|U(., 0)\|_{H^{s} x H^{s}}$ elegidos posteriormente. Por demostrar que: a. La aplicación P está bien definida por (9) para cualquier $\mathrm{T}>0 \mathrm{y} R>0$, es decir PU : $[0, \mathrm{~T}] \rightarrow H^{s} x H^{s}$.

b. Para cualquier T>0, la aplicación PU: $[0, \mathrm{~T}] \rightarrow H^{s} x H^{s}$ es continua, esto es, PU $\in C\left([0, T]: H^{s} x H^{s}\right)$ para todo $\mathrm{U} \in \mathrm{Y}(\mathrm{T}, \mathrm{R})$.

c. Existen $\mathrm{T}_{0}=\mathrm{T}_{0}\left(\|U(., 0)\|_{H^{s} x H^{s}}\right)>0$ y $\mathrm{R}_{0}=\mathrm{R}_{0}\left(\|U(., 0)\|_{H^{s} x H^{s}}\right)$ $>0$ tal que la aplicación $\mathrm{P}$ definida en $\mathrm{Y}\left(\mathrm{T}_{0}, \mathrm{R}_{0}\right)$ tiene rango $\mathrm{R}(\mathrm{P})$ contenido en $\mathrm{Y}\left(\mathrm{T}_{0}, \mathrm{R}_{0}\right)$.

En efecto:

a.(PU)(.,0) $=\mathrm{U}(., 0)-\int_{0}^{t} A^{-1} \partial_{x}[B U(., \tau)+F(U(., \tau))] d \tau$, aplicando el teorema 27 (PU)(.,t) $=\mathrm{U}(., 0)$ - $\int_{0}^{t} K * \partial_{x}[B U(., \tau)+F(U(., \tau))] d \tau$. Por hipótesis $U(., 0) \in H^{s} x H^{s}$ y si, $U(., t) \in H^{s} x H^{s}$ entonces $u(t) \in H^{s}$ y $v(t) \in H^{s}$. De $(4 *), F(U(., t)) \in H^{s} x H^{s}$ pues para $\mathrm{s} \geq 2$, es un álgebra de Banach, por lo tanto $v^{p+1}(t), u^{p+1}(t), u^{p}(t) v(t), u(t) v^{p}(t)$ y sus productos por escalares pertenecen a $H^{s}$. Por el teorema $28 \mathrm{~K}^{*} \partial_{x}$ $[\mathrm{BU}(., \tau)+\mathrm{F}(\mathrm{U}(., \tau))] \epsilon H^{s} x H^{s}$ y dado que $H^{s+1} x H^{s+1} \subseteq H^{s} x H^{s}$, se concluye que $\int_{0}^{t} K^{*} \partial_{x}[B U(., \tau)+F(U(., \tau))] d \tau \in H^{s} x H^{s}$. Luego $(\mathrm{PU})(., \mathrm{t}) \in H^{s} x H^{s}$ para cualquier $\mathrm{t} \in[0, \mathrm{~T}]$, lo que prueba que $\mathrm{P}$ está bien definida por (9).

b. Para $\mathrm{t}_{0} \in[0, \mathrm{~T}]$ supongamos $\mathrm{t}_{0}<\mathrm{t}$, entonces $\left\|P U(t)-P U\left(t_{0}\right)\right\|_{H^{s} x H^{s}}, \leq \int_{t_{0}}^{t}\left\|A^{-1} \partial_{x}[B U(., \tau)+F(U(., \tau))]\right\|_{H^{s} x H^{s}} d \tau \mathrm{de}$ los teorema 27 y 28 se tiene

$\left\|P U(t)-P U\left(t_{0}\right)\right\|_{H^{s} x H^{s}} \leq \int_{t_{0}}^{t}\left\|K^{*} \partial_{x}[B U(., \tau)+F(U(., \tau))]\right\|_{H^{s} x H^{s}} d \tau$

$\leq \mathrm{C} .\left(t-t_{0}\right) \sup _{t \leq \tau \leq t}\|B U(., \tau)+F(U(., \tau))\|_{H^{s} x H^{s}}$

Cuando $t \rightarrow t_{0}^{+}$la $_{0} \leq t \leq t$ expresión converge a cero. Esto prueba la continuidad de PU por la derecha de $t_{0}$. La continuidad por la izquierda de $\mathrm{t}_{0}$ se prueba de manera análoga. Luego $\mathrm{PU}$ es continua en $\mathrm{t}_{0}$

c. Sean $\mathrm{T}_{0}>0$ y $\mathrm{R}_{0}>0$ y $\mathrm{U}(\mathrm{t}) \in \mathrm{Y}\left(\mathrm{T}_{0}, \mathrm{R}_{0}\right)$, para $0 \leq \mathrm{t} \leq \mathrm{T}_{0}$ y el teorema de Bochner $\|P U(., t)-U(., 0)\|_{H^{s} x H^{s}} \leq$ $\mathrm{y} \int_{0}^{t}\left\|K^{*} \partial_{x}[B U(., \tau)+F(U(., \tau))]\right\|_{H^{s} x H^{s}} d \tau$ el teorema 28 , $\|P U(., t)-U(., 0)\|_{H^{s} x H^{s}} \leq \mathrm{C}, \int_{0}^{t}\left(\|B U(., \tau)\|_{H^{s} x H^{s}}+\|F(U(., \tau))\|_{H^{s} x H^{s}}\right) d \tau$ como $\mathrm{F}(\mathrm{U}(., \mathrm{t}))=$

$\left(a_{3} \frac{v^{p+1}}{p+1}+\frac{u^{p+1}}{p+1}+a_{4}\left(u^{p} v\right), a_{3} \frac{u^{p+1}}{p+1}+\frac{v^{p+1}}{p+1}+a_{4}\left(u v^{p}\right)\right)(t) \leq$

$\frac{1}{p+1}\left[\left(1+\left|a_{3}\right|\right)\left(\|v\|_{s}^{p+1}+\|u\|_{s}^{p+1}\right)+(p+1)\left|a_{4}\right|\left(\|u\|_{s}^{p}\|v\|_{s}+\|u\|_{s}\|v\|_{s}^{p}\right)\right]$

$\|P U(., t)-U(., 0)\|_{H^{s} x H^{s}} \leq \mathrm{M}$

$\int_{0}^{t}\left[\|U(., \tau)\|_{H^{s} x H^{s}}+\|v\|_{s}^{p+1}+\|u\|_{s}^{p+1}+\|u\|_{s}^{p}\|v\|_{s}+\|u\|_{s}\|v\|_{s}^{p}\right] d \tau$

donde $\mathrm{M}=\operatorname{máx}\left\{C|B|, \frac{C\left(1+\left|a_{3}\right|\right)}{p+1}, C\left|a_{4}\right|\right\}$ Por definición

del espacio $\mathrm{Y}\left(\mathrm{T}_{0}, \mathrm{R}_{0}\right):\|U(., \tau)\|_{H^{x}, H^{\prime}} \leq R_{0}+\|U(., 0)\|_{H^{\prime} \times H^{\prime}}, \forall \tau \in\left[0, T_{0}\right] .(11)$ entonces, $\|u(\tau)\|_{s} \leq\|U(., \tau)\|_{H^{s} x H^{s}} \leq\|U(., \tau)-U(., 0)\|_{H^{s} x H^{s}}+\|U(., \tau)\|_{H^{s} x H^{s}}$ $\leq \mathrm{R}_{0}+\|U(., 0)\|_{H^{s} x H^{s}}, \forall \tau \in\left[0, T_{0}\right]$

Del mismo modo, $\|v(\tau)\|_{s} \leq\|U(., \tau)\|_{H^{s} x H^{s}} \leq \mathrm{R}_{0}+$

$\|U(., 0)\|_{H^{s} x H^{s}}, \forall \tau \in\left[0, T_{0}\right]$.

Reemplazando (11),(12),(13) en (10) obtenemos $\|P U(., t)-U(., 0)\|_{H^{s} x H^{s}} \leq \mathrm{M}\left[\mathrm{R}_{0}+\|U(., 0)\|_{H^{s} x H^{s}}+4\right]$ $\left(R_{0}+\|U(., 0)\|_{H^{s} \times H^{s}}\right)^{p+1} \mathrm{t}$, para todo $\mathrm{t} \leq \mathrm{T}_{0}$ Luego $\|P U(., t)-U(., 0)\|_{H^{s} x H^{s}}$ $\leq \operatorname{MT}_{0}\left(R_{0}+\|U(., 0)\|_{H^{s} x H^{s}}\right)\left(1+4\left(R_{0}+\|U(., 0)\|_{H^{s} x H}\right)^{p}\right)$. Eligiendo $\mathrm{R}_{0}=\|U(., 0)\|_{H^{s} x H^{s}} \mathrm{y}_{0}=\frac{1}{2 M\left(1+2^{p+2}\|U(., 0)\|_{H^{s} H^{s}}^{p}\right)}$, se tiene $\|P U(., t)-U(., 0)\|_{H^{s} x H^{s}} \mathrm{R}_{0}$, para todo $\mathrm{t} \epsilon\left[0,{ }_{H^{s} H^{s}}\right]$. Por consiguiente, $\sup _{0 \leq t \leq T_{0}}\|P U(., t)-U(., 0)\|_{H^{s} x H^{s}} \leq \mathrm{R}_{0,}$ asi
$\mathrm{R}(\mathrm{P}) \subseteq Y\left(T_{0} R_{0}\right)$. Segunda etapa.

Para demostrar que existe $\bar{T} \in\left[0, T_{0}\right]$ y $\bar{R} \in\left[0, R_{0}\right]$ tal que la aplicación P: $Y(\bar{T}, \bar{R}) \rightarrow Y(\bar{T}, \bar{R})$ es una contracción. En efecto si $\mathrm{U}=\left(\mathrm{u}_{1}, \mathrm{v}_{1}\right), \mathrm{V}=\left(\mathrm{u}_{2}, \mathrm{v}_{2}\right) \in \mathrm{Y}\left(\mathrm{T}_{0} \mathrm{R}_{0}\right)$, aplicando el teorema 27 y $28 \leq\|P U(., t)-P V(., t)\|_{H^{s} x H^{s}}$ $C \int_{0}^{t}|B|\|V(., \tau)-U(., \tau)\|_{H^{s} x H^{s}} d \tau+\mathrm{C}$ 


$$
\begin{aligned}
& \int_{0}^{t}|B|\|F(V(., \tau))-F(U(., \tau))\|_{H^{s} x H^{s}} d \tau \\
& \text { Pero }\|F(V(., \tau))-F(U(., \tau))\|_{H^{s} x H^{s}} \leq \\
& \frac{1}{p+1}\left[\mid a_{3}\left\|v_{2}^{p+1}-v_{1}^{p+1}\right\|_{s}+\left\|u_{2}^{p+1}-u_{1}^{p+1}\right\|_{s}\right]+ \\
& \frac{1}{p+1}\left[\left|a_{4}\right|(p+1)\left\|u_{2}^{p} v_{2}-u_{1}^{p} v_{1}\right\|_{s}+\left|a_{3}\right|\left\|u_{2}^{p+1}-u_{1}^{p+1}\right\|_{s}\right] \\
& +\frac{1}{p+1}\left[\left\|v_{2}^{p+1}-v_{1}^{p+1}\right\|_{s}+\left|a_{4}\right|(p+1)\left\|u_{2} v_{2}^{p}-u_{1} v_{1}^{p}\right\|_{s}\right] .
\end{aligned}
$$

Aplicando la norma en $H^{s}$ el hecho de que $H^{s}$ es un álgebra de Banach para $\mathrm{s} \geq 2$,

$\left\|p_{2}^{p+1}-v_{1}^{p+1}\right\|_{s} \leq C\left\|v_{2}-v_{1}\right\|_{s} \sum_{i=0}^{p}\left\|v_{2}\right\|_{s}^{p-i}\left\|v_{1}\right\|_{s}^{i}$,

$\left\|u_{2}^{p+1}-u_{1}^{p+1}\right\|_{s} \leq C\left\|u_{2}-u_{1}\right\|_{s} \sum_{i=0}^{p}\left\|u_{2}\right\|_{s}^{p-i}\left\|u u_{1}\right\|_{s}^{i}$

$\left\|u_{2}^{p} v_{2}-u_{1}^{p} v_{1}\right\|_{s}=\left\|u_{2}^{p} v_{2}-u_{1}^{p} v_{1}+u_{1}^{p} v_{2}-u_{1}^{p} v_{2}\right\|_{s} \leq C\left\|_{2}\right\|_{s}\left\|_{2}-u_{1}\right\|_{s}^{p-1} \sum_{i=0}^{p-1}\left\|u_{2}\right\|_{s}^{p-1-i}\left\|u_{1}\right\|_{s}^{i}$

$+C\left\|u_{1}\right\|_{s}^{p}\left\|v_{2}-v_{1}\right\|_{s}(17)$
$\left\|u_{2} v_{2}^{p}-u_{1} v_{1}^{p}\right\|_{s} \leq C\left\|u_{2}\right\|_{s}\left\|v_{2}-v_{1}\right\|_{s} \sum_{i=0}^{p-1}\left\|v_{2}\right\|_{s}^{p-1-i}\left\|v_{1}\right\|_{s}^{i}+$

$C\left\|v_{1}\right\|_{s}^{p}\left\|u_{2}-u_{1}\right\|_{s}$

Del mismo modo

$\left\|v_{1}(\tau)\right\|_{s} \leq\|V(., \tau)\|_{H^{s} x H^{s}} \leq R_{0}+\|U(., \tau)\|_{H^{s} x H^{s}}, \forall \tau \in\left[0, T_{0}\right] \ldots$

$\left\|u_{2}(\tau)\right\|_{s} \leq\|V(., \tau)\|_{H^{s} X^{s}} \leq R_{0}+\|U(., \tau)\|_{H^{s} x H^{s}}, \forall \tau \in\left[0, T_{0}\right]$

De las desigualdades (16),(17), y (18), se obtiene

$\left\|v_{2}^{p+1}-v_{1}^{p+1}\right\|_{s} \leq C\left\|v_{2}-v_{1}\right\|_{s} \sum_{i=0}^{p}\left(R_{0}+\|U(., 0)\|_{H^{s} x H^{s}}\right)^{p-i+i}$

$=\mathrm{C}\left\|v_{2}-v_{1}\right\|_{s}(\mathrm{p}+1)\left(R_{0}+\|U(., 0)\|_{H^{s} \times H^{s}}\right)^{p}$

$\left\|u_{2}^{p+1}-u_{1}^{p+1}\right\|_{s} \leq C\left\|u_{2}-u_{1}\right\|_{s}(p+1)\left(R_{0}+\|U(., 0)\|_{H^{s} x H^{s}}\right)^{p}$ (20)

$\left\|u_{2}^{p} v_{2}-u_{1}^{p} v_{1}\right\|_{s} \leq C\|V(., \tau)-U(., \tau)\|_{H^{\prime}: H^{\prime}}(p+1)\left(R_{0}+\|U(., 0)\|_{H^{\prime}: H^{\prime}}\right)^{p}$

$\left\|u_{2} v_{2}^{p}-u_{1} v_{1}^{p}\right\|_{s} \leq C\|V(., \tau)-U(., \tau)\|_{H^{s} \times H^{s}}(p+1)\left(R_{0}+\|U(., 0)\|_{H^{x} x^{s}}\right)^{p}$

Reemplazando (19), (20), (21) y (22) en (15) obtenemos $\| F(V(., \tau))-F\left(U(., \tau)\left\|_{H^{\prime}: H^{\prime}} \leq 3 k_{1}\right\| V(., \tau)-U(., \tau) \|_{H^{\prime} \times H^{\prime}}(p+1)\left(R_{0}+\|U(., 0)\|_{H^{\prime} \times H^{\prime}}\right)^{p}\right.$, (23)

Donde $\mathrm{k}_{1}=\operatorname{máx}\left\{\mathrm{Cla}_{3}\left|, \mathrm{C}, \mathrm{Cla}_{4}\right|\right\}$. Sustituyendo (23) en (14), $\mathrm{k}_{2}=$ máx $\left\{\mathrm{C} \mid \mathrm{BI}, 3 \mathrm{k}_{1} \mathrm{C}\right\}$ y para todo $\mathrm{t} \epsilon\left[0, \mathrm{~T}_{0}\right]$ :

$\|P U(., t)-P V(., t)\|_{H^{s} x H^{s}} \leq k_{2}\left[1+\left(R_{0}+\|U(., 0)\|_{H^{s} x H^{s}}\right)^{p}\right] T_{0} d(U, V)$ Eligiendo $R_{0}=\bar{R}=\|U(., 0)\|_{H^{s} x x^{s}} y \forall t \in\left[0, T_{0}\right]$ se tiene $\|P U(., t)-P V(., t)\|_{H^{s} x H^{s}} \leq k_{2}\left[1+2^{p}\|U(., 0)\|_{H^{s} x H^{s}}^{p}\right] T_{0} d(U, V)$. Aplicar el supremo en $\left[0, \mathrm{~T}_{0}\right], \mathrm{d}(\mathrm{PU}, \mathrm{PV})=$ $\sup _{0 \leq t \leq T_{0}}\|P U(., t)-U(., 0)\|_{H^{s} x H^{s}} \leq k_{2}\left[1+2^{p}\|U(., 0)\|_{H^{s} x H^{s}}^{p}\right] T_{0} d(U, V)$. Como $k_{2}\left[1+2^{p}\|U(., 0)\|_{H^{s} x H^{s}}^{p}\right] T_{0} \rightarrow 0$ cuando $\mathrm{T}_{0} \rightarrow 0^{+}$, de la definición de limite existe $\bar{T}=\bar{T}\left(\|U(., 0)\|_{H^{s} \times H^{s}}\right)$ tal que

$0<<\mathrm{T}_{0}<\delta=\delta\left(\|U(., 0)\|_{H^{s} x H^{s}}\right), \delta(x)=\frac{\lambda}{k_{2}\left(1+2^{p} x^{p}\right)}$

Así $\mathrm{d}(\mathrm{PU}, \mathrm{PV}) \leq \lambda \mathrm{d}(\mathrm{U}, \mathrm{V})$, con $0 \ll 1$, lo que muestra que $\mathrm{P}$ es una contracción.

Por el teorema del punto fijo de Banach, existe una única $\mathrm{U} \in \mathrm{Y}(\bar{T}, \bar{R}) \subseteq C\left([0, \bar{T}]: H^{s} x H^{s}\right)$ tal que $\mathrm{PU}=\mathrm{U}$, es decir, $\mathrm{PU}(\mathrm{x}, \mathrm{t})=\mathrm{U}(\mathrm{x}, \mathrm{t})=\mathrm{U}(\mathrm{x}, 0)-\int_{0}^{t} k^{*} \partial_{x}[B U(x, \tau)+F(U(x, \tau))] d \tau$, para todo $\mathrm{t} \in[0, \bar{T}]$, lo cual muestra la existencia de la ecuación integral $(\mathrm{EI}) \operatorname{er}(\overline{\mathrm{Y}}, \bar{R})$

A continuación probaremos que la función $\mathrm{U}$, solución de la ecuación integral (EI), es la única solución de (3).

Teorema 31. Sea $\mathrm{U}_{0}=\left(\mathrm{u}_{0}, \mathrm{v}_{0}\right) \in H^{s} x H^{s}, \mathrm{~s} \geq 2, \mathrm{~s} \in \mathrm{Z}$, $\mathrm{a}_{1}, \mathrm{a}_{2} \mathrm{a}_{3} \mathrm{a}_{4}$ números reales con $\mathrm{a}_{2}>0<\mathrm{a}_{1}<1$ y $\mathrm{p}$ un entero $\geq 1$ Entonces existen $>0$ y un par de funciones $(\mathrm{u}, \mathrm{v})$ $\in C\left([0, \bar{T}]: H^{s}\right)^{2}$ tal que $\left(\partial_{\mathrm{t}} \mathrm{u}, \partial_{\mathrm{t}} \mathrm{v}\right) \in C\left([0, \bar{T}]: H^{s}\right)^{2}$ y (u, v) satisface (4).

Demostración. Por demostrar que la función U, solución única de la ecuación integral (EI) es solución del problema de valor inicial (3) y que $\partial_{\mathrm{x}} \mathrm{U}(\mathrm{x}, \mathrm{t})$ existe. En efecto consideremos $\mathrm{U}(\mathrm{x}, \mathrm{t})=\mathrm{U}(\mathrm{x}, 0)-\int_{0}^{t} \partial_{x} k *[B U(x, \tau)+F(U(x, \tau))] d \tau$. (25)

Hagamos $\mathrm{U}_{\mathrm{L}}(\mathrm{x}, 0)=\mathrm{U}(\mathrm{x}, 0)$ y $\mathrm{U}_{\mathrm{p}}(\mathrm{x}, \mathrm{t})=-$

$\int_{0}^{t} \partial_{x} k *[B U(x, \tau)+F(U(x, \tau))] d \tau$. Luego, $\mathrm{U}_{\mathrm{L}}(\mathrm{x}, \mathrm{t}) \quad$ es solución del problema lineal $\mathrm{A}_{\mathrm{t}} \mathrm{U}_{\mathrm{L}}(\mathrm{x}, \mathrm{t})+\mathrm{BU}_{\mathrm{L}}(\mathrm{x}, \mathrm{t})=0$, $\mathrm{U}_{\mathrm{L}}(\mathrm{x}, 0)=\mathrm{U}(\mathrm{x}, 0)=\left(\mathrm{u}_{0}, \mathrm{~V}_{0}\right)$. , y demostraremos que $\mathrm{U}_{\mathrm{p}}(\mathrm{x}, \mathrm{t})$ es solución de $\mathrm{A} \partial_{\mathrm{t}} \mathrm{U}_{\mathrm{p}}(\mathrm{x}, \mathrm{t})+\mathrm{B} \partial_{\mathrm{x}} \mathrm{U}_{\mathrm{p}}(\mathrm{x}, \mathrm{t})+\partial_{\mathrm{x}} \mathrm{F}\left(\mathrm{U}_{\mathrm{p}}(\mathrm{x}, \mathrm{t})\right)=$ $0, \mathrm{U}_{\mathrm{p}}(\mathrm{x}, 0)=0$. Sea $\mathrm{h}>0$ tal que $\mathrm{t}+\mathrm{h} \quad \in[0, \bar{T}]$, así $\frac{U_{P}(x, t+h)-U_{P}(x, t)}{h}=-\frac{1}{h} \int_{t}^{t+h} \partial_{x} k *\left[B U_{P}(x, \tau)+F(U(x, \tau))\right] d \tau$ (26)

Por el teorema del valor medio para integrales de Bochner en el intervalo $[\mathrm{t}, \mathrm{t}+\mathrm{h}]$ con $\mathrm{t}_{\mathrm{h}} \in[\mathrm{t}, \mathrm{t}+\mathrm{h}]$ y las propiedades de limite cuando $\mathrm{h} \rightarrow 0^{+}$a (26), se tiene $\partial_{t}^{+} U_{P}(x, t)=-\partial_{x} k *\left[B U_{P}(x, t)+F\left(U_{P}(x, t)\right)\right]$ En forma análoga, para $\mathrm{h}<0 \quad \partial_{t}^{-} U_{P}(x, t)=-\partial_{x} k *\left[B U_{P}(x, t)+F\left(U_{P}(x, t)\right)\right]$. Se concluye que $\partial_{t}^{+} U_{P}(x, t)=\partial_{t}^{-} U_{P}(x, t)$. Luego existe $\partial_{\mathrm{t}} \mathrm{U}_{\mathrm{p}}(\mathrm{x}, \mathrm{t})$, integrando 0de 0 a t, $\mathrm{U}_{\mathrm{p}}(\mathrm{x}, \mathrm{t})=-$

$\int_{0}^{t} A^{-1}\left[B \partial_{x} U_{P}(x, \tau)+\partial_{x} F\left(U_{P}(x, \tau)\right)\right] d \tau$, así, existe $\mathrm{U}_{\mathrm{p}}(\mathrm{x}, \mathrm{t})$ $\mathrm{y}$ es solución de la ecuación (3). Del mismo modo se prueba que $\mathrm{U}=\mathrm{U}_{\mathrm{L}}+\mathrm{U}_{\mathrm{p}}$ es solución de (3). Por el Teorema de Inmersión de Sobolev se tiene $H^{s} x H^{s}-\mathrm{C}_{\infty} \mathrm{xC}$ de ahí que $\partial_{\mathrm{t}} \mathrm{U}(., \mathrm{t}) \in C\left([0, \bar{T}]: H^{s} x H^{s}\right)$. Así, $\partial_{\mathrm{t}} \mathrm{U}(., \mathrm{t})$ existe y está dado por $\partial_{t} U(., t)=\partial_{t} P U(., t)=-A^{-1} \partial_{x}[B U(., t)+F(U(., t))]$.

que es una función continua en $\mathrm{t} \in[0, \bar{T}]$ con valores en $H^{s} x H^{s}$. Con esto se prueba la existencia local de la solución de (3) y la unicidad en . Queda por probar la unicidad en $C\left([0, \bar{T}]: H^{s} x H^{s}\right)$.

\section{Unicidad de la solución local.}

La unicidad de la solución local del problema (3) será una consecuencia inmediata del siguiente teorema

Teorema 32. Sean $\mathrm{U}(., 0), \mathrm{V}(., 0) \in$ con $\mathrm{s} \geq 2$. Entonces existen $\mathrm{T}>0$ y $\mathrm{U}, \mathrm{V} \in C\left([0, T]: H^{s} x H^{s}\right)$ soluciones de (3) tales que $\mathrm{U}(\mathrm{x}, 0)=\mathrm{U}(0), \mathrm{V}(\mathrm{x}, 0)=\mathrm{V}(0) \mathrm{y}$

$\|U(., t)-V(., t)\|_{H^{s} x H^{s}} \leq\|U(., 0)-V(., 0)\|_{H^{s} x H^{s}} e^{C t}, \forall t \in[0, T](27)$

Demostración. Por el teorema 31, existen $\left.\left.\bar{T}_{U(0)}\right\rangle 0 y \bar{T}_{V(0)}\right\rangle 0$ tales que $\mathrm{U} \in C\left(\left[0, \bar{T}_{U(0)}\right]: H^{s} x H^{s}\right)$ y $\mathrm{V} \in C\left(\left[0, \bar{T}_{V(0)}\right]: H^{s} x H^{s}\right)$, con $\mathrm{s} \geq 0, \mathrm{U}=\left(\mathrm{u}_{1}, \mathrm{v}_{1}\right)$ y $\mathrm{V}=\left(\mathrm{u}_{2}, \mathrm{v}_{2}\right)$ que satisfacen las ecuaciones

$$
\begin{aligned}
& \mathrm{U}(\mathrm{x}, \mathrm{t})=\mathrm{U}(\mathrm{x}, 0)-\int_{0}^{t} A^{-1}\left[B \partial_{x} U(x, \tau)+\partial_{x} F(U(x, \tau))\right] d \tau \\
& \mathrm{V}(\mathrm{x}, \mathrm{t})=\mathrm{V}(\mathrm{x}, 0)-\int_{0}^{t} A^{-1}\left[B \partial_{x} V(x, \tau)+\partial_{x} F(V(x, \tau))\right] d \tau,
\end{aligned}
$$


para todo te $[0, \mathrm{~T}]$, siendo $\mathrm{T}=$ mín. $\left\{\bar{T}_{U(0)}, \bar{T}_{V(0)}\right\}$ Entonces $\|U(., t)-V(., t)\|_{H^{\prime} \rightarrow H^{\prime}} \leq\|U(., 0)-V(., 0)\|_{H^{\prime} H^{\prime}}+C_{1}|B| \int_{0}^{t}\|U(., \tau)-V(., \tau)\|_{H^{\prime} H^{\prime}} d \tau$ $+\mathrm{C}_{2} \int_{0}^{t}\|F(U(., \tau)-V(., \tau))\|_{H^{s} x H^{s}} d \tau$.

Acotando, para ello usamos las ecuaciones (15), (16), (17), (18) y como $H^{s}$ es un álgebra de Banach para $s \geq 2$ y por equivalencia de normas se tiene:

$\|F(U(., \tau))-F(V(., \tau))\|_{H^{s} x H^{s}} \leq C_{3} M\left\|\left(u_{1}, v_{1}\right)-\left(u_{2}, v_{2}\right)\right\|_{H^{s} \times H^{s}}$ Donde $\mathrm{M}=$ máx $\left\{\left(1+\left|a_{3}\right|\right) N^{p},\left|a_{4}\right| N^{p},\left|a_{4}\right| N^{p} \mathrm{p}\right\}$ y $\mathrm{N}=$ máx \{ $\left.\sup _{[0, T]}\|U(., \tau)\|_{H^{s} \times H^{s}} \sup _{[0, T]}\|V(., \tau)\|_{H^{s} \times H^{s}}\right\}$.Sustituyendo en la ecuación (28) y haciendo $\mathrm{C}=\mathrm{C}_{1}|B|+\mathrm{C}_{2} \mathrm{r}$, Obtenemos $\|U(., \tau)-V(., \tau)\|_{H^{s} x H^{s}} \leq\|U(., 0)-V(., \tau)\|_{H^{s} x H^{s}}+\mathrm{C}$

\section{$\int_{0}^{t}\|U(., \tau)-V(., \tau)\| d \tau$}

Aplicando la desigualdad de Gronwall se tiene para todo tє $[0, \mathrm{~T}]\|U(., \tau)-V(., \tau)\|_{H^{s} x H^{s}} \leq\|U(., 0)-V(., 0)\|_{H^{s} x H^{s}} e^{C T}$. La que prueba el teorema.

Corolario 3 . Si U $(0) \in H^{s} x H^{s}$ con $\mathrm{s} \geq 0$, existen $\bar{T}=\bar{T}\left(\|U(0)\|_{H^{s} H^{*}}\right)$ $>0$ y $\mathrm{U} \in C\left([0, \bar{T}]: H^{s} x H^{s}\right)$ con $\partial_{\mathrm{t}} \mathrm{U} \in C\left([0, \bar{T}]: H^{s} x H^{s}\right)$ única solución de (3).

Demostración Sean U y V dos soluciones de (3), con datos iniciales $\mathrm{U}(0)=\mathrm{V}(0)$, en (27), así $\|U(., \tau)-V(., \tau)\|_{H^{x} \times H^{s}}=$ 0 , para todo te $[0, \bar{T}]$. Esto prueba la unicidad local en $C\left([0, \bar{T}]: H^{s} x H^{s}\right)$.

\section{Dependencia continua de la solución local respecto del} dato inicial.

Veamos que la solución depende continuamente del dato inicial.

Teorema 34. Sean $\mathrm{U}_{\mathrm{n}}(0), \mathrm{U}(0) € H^{s} x H^{s} \operatorname{con} \mathrm{U}_{\mathrm{n}}(0) \rightarrow \mathrm{U}(0)$

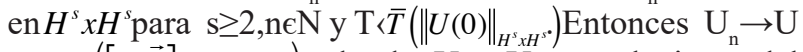
en $C\left([0, \vec{T}]: H^{s} x H^{s}\right)$ donde $\mathrm{U}_{\mathrm{n}} \mathrm{y}$ U son soluciones del problema (3) respecto a los datos iniciales $\mathrm{U}_{\mathrm{n}}(0)$ y $\mathrm{U}(0)$.

Demostración. Dado $T<\bar{T}\left(\|U(0)\|_{H^{s} x_{H}^{s}}\right)$, por demostrar que si $\left\{U_{n}(0)\right\}_{n>0} \in H^{s} x H^{s}$ es una sucesión tal que $\lim _{n \rightarrow+\infty} U_{n}(0)$ $=\mathrm{U}(0)$ en $H^{s} x H^{s}$, entonces existe $n_{0} \in N$ de manera que para cualquier $\mathrm{n}>$ y $\mathrm{T}<\bar{T}\left(\|U(0)\|_{H^{s} \times H^{s}}\right), U_{n} \rightarrow \mathrm{U}$ en $C\left([0, \bar{T}]: H^{s} x H^{s}\right)$ o $\lim _{n \rightarrow+\infty} \sup _{t \in[0, T]}\left\|U_{n}(t)-U(t)\right\|_{H^{s} x H^{s}}=0$, por el teorema 31 y (11); $\bar{T}$ está definida por

$\left.\bar{T}(U(0))=\left(R_{0}+\|U(0)\|_{H^{s} x H^{s}}\right) \in\right] 0,+\infty[$ función continua en $\mathrm{U}(0)$, si $\mathrm{U}_{\mathrm{n}}(0) \rightarrow \mathrm{U}(0)$ en $H^{s} x H^{s}$, entonces

$\left|\bar{T}\left(U_{n}(0)\right)-\bar{T}(U(0))\right| \leq\left\|U_{n}(0)-U(0)\right\|_{H^{s} x H^{s}}$. Así, T es una función continua en $\mathrm{U}(0)$, y por lo tanto existe tal que $\mathrm{T}<\bar{T}\left(\|U(0)\|_{H^{s} \times H^{s}}\right)$ para todo $\mathrm{n} \geq n_{0}$; de este modo $U_{n}$ está definida en $[0, T]$ para todo $\mathrm{n} \geq$.

Sea $\epsilon \mathrm{Y}(\mathrm{T}, \mathrm{R}),\left\|U_{n}(t)\right\|_{H^{s} x H^{s}} \leq R_{0}+\left\|U_{n}(0)\right\|_{H^{s} x H^{s}}=\mathrm{R}_{0}+\mathrm{M}$, donde $\mathrm{M}=\sup _{t \in[0, T]}\left\|U_{n}(0)\right\|_{H^{s} x H^{s}}$.
Por el teorema $31\left\|U_{n}(t)-U(t)\right\|_{H^{s} x H^{s}} \leq\left\|U_{n}(0)-U(0)\right\|_{H^{s} x H^{s}}+$ $\mathrm{C}_{1}|\mathrm{~B}| \int_{0}^{t}\left\|U_{n}(., \tau)-U(., \tau)\right\|_{H^{s} x H^{5}} d \tau+\mathrm{C}_{2}$

$\int_{0}^{t}\left\|F\left(U_{n}(., \tau)\right)-F(U(., \tau))\right\|_{H^{x} x^{\prime}} d \tau$.

Por el teorema $32, F\left(U_{n}(., \tau)\right)-F(U(., \tau))\left\|_{H^{\prime} \times H^{*}} \leq M\right\| U_{n}(., \tau)-U(., \tau) \|_{H^{\prime} \times t h}$, se tiene $\left\|U_{n}(., t)-U(., t)\right\|_{H^{s} x H^{s}} \leq\left\|U_{n}(0)-U(0)\right\|_{H^{s} x H^{s}}+\mathrm{C}$

$\int_{0}^{t}\left\|U_{n}(., \tau)-U(., \tau)\right\|_{H^{s} x H^{s}} d \tau \quad$. Aplicando la desigualdad de Gronwall, $\left\|U_{n}(., t)-U(., t)\right\|_{H^{s} x H^{s}} \leq\left\|U_{n}(0)-U(0)\right\|_{H^{s} x H^{s}} e^{C T}, \mathrm{t}$ 〈 $\mathrm{T}$. Al aplicar el supremo sobre $\mathrm{t} \epsilon[0, \mathrm{~T}]$ y límite cuando $\mathrm{n} \rightarrow+\infty$ se obtiene el resultado.

De esta manera queda probada la dependencia continua de la solución respecto del dato inicial, con lo cual el problema está bien formulado localmente.

\section{Resultados y discusión}

En este trabajo, se estudió el problema de Cauchy para el sistema dispersivo no lineal de tipo BBM. El resultado de la existencia y unicidad de la solución local para la ecuación integral asociada al sistema, se ha obtenido al aplicar el teorema del punto fijo de Banach; las proposiciones 29, 30; los teoremas 31,32 y el corolario 33; el teorema 34 garantiza que la solución local depende continuamente del dato inicial.

\section{Conclusiones}

Como se vio, el problema de Cauchy asociado al sistema dispersivo no lineal de tipo BBM (P) está bien formulado localmente. Se recomienda trabajar en el comportamiento asintótico de la ecuación (P), que consiste en estudiar si es que la solución global del problema (P) decae o explota cuando $\mathrm{t} \rightarrow+\infty$.

\section{Literatura citada}

Benjamin, T.B.; Bona, J.L. y Mahony, J.J. 1972. Model equations for long waves in nonlinear dispersive systems. Philos. Trans. Royal Soc. London, series A, (272), 4748. 